\title{
Incidence of Venous Thromboembolism in Asian Populations: A Systematic Review
}

\author{
Lai Heng Lee Lexander Gallus $^{4,5}$ Ravul Jindal ${ }^{6}$ \\ ${ }^{1}$ Department of Haematology, Singapore General Hospital, Singapore \\ 2 Yong Loo Lin School of Medicine, National University of Singapore, \\ Singapore \\ ${ }^{3}$ Duke-NUS Medical School, Singapore \\ 4 Department of Haematology, Flinders University, Adelaide, SA, Australia \\ ${ }^{5}$ Flinders Medical Centre, Adelaide, SA, Australia \\ 6 Vascular Surgery, Fortis Hospital, Mohali, India \\ ${ }^{7}$ National Clinical Research Center of Respiratory Diseases, \\ Department of Pulmonary and Critical Care Medicine, \\ China-Japan Friendship Hospital, Beijing, China \\ ${ }^{8}$ Department of Respiratory Medicine, Capital Medical University, \\ Beijing, China \\ ${ }^{9}$ Department of Internal Medicine (Cardiology Section), \\ National Taiwan University Hospital, Taipei, Taiwan \\ 10 Graduate Institute of Medical Education and Bioethics, College of \\ Medicine, National Taiwan University, Taipei, Taiwan
}

Chen Wang ${ }^{7,8}$ Chau-Chung $\mathrm{Wu}^{9,10}$

\author{
Address for correspondence Prof. Lai Heng Lee, MBBS, MMed, FRCP, \\ Department of Haematology, Singapore General Hospital, Outram \\ Road, Singapore 169608, Singapore \\ (e-mail: lee.lai.heng@singhealth.com.sg).
}

Thromb Haemost 2017;117:2243-2260.

\begin{abstract}
Keywords

- venous thromboembolism

- deep vein thrombosis

- pulmonary embolism

- epidemiology

- Asian population

Introduction Despite a marked recent increase in the number of publications describing the incidence of venous thromboembolism (VTE) in Asia, and especially in mainland China, Hong Kong, Taiwan, Korea, Japan and Singapore, there remains a lack of consensus on the true risks, and trends over time, to inform appropriate clinical practice. The purpose of this systematic review was therefore to examine evidence about the incidence of symptomatic VTE in Asia.

Methods Databases were searched for studies from Asia, published between January 1995 and February 2016, on the incidence of symptomatic VTE, deep vein thrombosis (DVT) or pulmonary embolism. Review of eligible studies was conducted independently by two reviewers. Data were extracted on incidence, predispositions and recurrence of VTE.

Results One thousand nighty-five studies were identified, of which 73 were eligible for full text review and data extraction. Three population-wide estimates of VTE rates identified from Korea, Taiwan and Hong Kong reported annual incidences of 13.8, 15.9 and 19.9 per 100,000 , respectively. Nine studies of Asian hospital registries or databases reported VTE rates ranging from 11 to 88 cases per 10,000 admissions. Population-based estimates of post-surgical DVT rates ranged from 0.15 to $1.35 \%$. Age was a significant risk factor for VTE in all population groups.

Conclusion Population-wide incidence estimates in Asia were approximately 15 to $20 \%$ of the levels recorded in western countries but have increased over time. It is anticipated this synthesis of evidence on the incidence of VTE and its predisposing factors will increase awareness about VTE in Asian populations.
\end{abstract}

received

February 24, 2017

accepted after revision
September 7, 2017

Copyright () 2017 Schattauer 


\section{Introduction}

Until recently, there has been a relative lack of research into the incidence of venous thromboembolism (VTE) in Asian populations, ${ }^{1}$ perhaps partly due to early impressions that VTE was rare in Asia. ${ }^{2}$ However, the past 15 years have seen a marked increase in the number of publications, especially from mainland China, Hong Kong, Taiwan, Korea, Japan and Singapore. Most of these studies have reported significant VTE rates in the respective Asian populations, although reported rates were generally lower than those in western populations where VTE has an estimated annual incidence of 1 per 1,000 per annum, which makes VTE a major health issue. Although VTE is ranked the third most frequent cause of cardiovascular death in Western countries, ${ }^{3}$ there remains a lack of acknowledgement of this condition in Asian populations. This systematic review aims to encapsulate the latest findings from Asia and raise awareness of VTE as an important condition in this region.

The effective management of symptomatic deep vein thrombosis (DVT) and pulmonary embolism (PE) requires early confirmation of clinically suspected disease. In turn, early confirmation is reliant on both clinicians and the public having a high local awareness of these disorders. A recent global survey indicated that clinical awareness regarding
VTE is generally low in Asian countries. ${ }^{4}$ Since the nations that comprise Asia account for more than half the world's population, such low local awareness about VTE is likely to add considerably to the avoidable burden from this disease.

The purpose of this systematic review is to examine evidence about the incidence of symptomatic VTE, reported between January 1995 and February 2016, in publications from East Asia. The focus is on population-based and other studies of VTE incidence, temporal trends in reported VTE rates, and the contributions of age and other major predisposing conditions. The results may help increase awareness about the importance of VTE in Asian populations.

Existing Asian VTE guidelines are summarized in - Table 1. The present synthesis of evidence on the incidence of VTE and predisposing factors may inform future refinements and updates of VTE-related guidelines in Asia.

\section{Methods}

For the purposes of this study only symptomatic VTE was considered and Asian populations were defined as people from the geographical regions of East Asia including China (including Hong Kong and Macau), Taiwan, Mongolia, Korea (North and South) and Japan, Southeast Asia including Vietnam, Malaysia, Thailand, Indonesia, Singapore and

Table 1 List of Asian guidelines and expert recommendations on prevention and/or management of venous thromboembolism (VTE)

\begin{tabular}{|c|c|c|c|}
\hline Guideline title & Year & Reference & \\
\hline $\begin{array}{l}\text { Asian venous thromboembolism } \\
\text { guidelines: updated } \\
\text { recommendations for the prevention } \\
\text { of venous thromboembolism }\end{array}$ & 2017 & Int Angiol 2017;36(1):1-20 & 68 \\
\hline $\begin{array}{l}\text { Management of venous thromboem- } \\
\text { bolisms: part I. The consensus for } \\
\text { deep vein thrombosis }\end{array}$ & 2016 & Acta Cardiol Sin 2016;32(1):1-22 & 69 \\
\hline $\begin{array}{l}\text { Prevention of venous thromboembo- } \\
\text { lism, 2nd edition: Korean Society of } \\
\text { Thrombosis and Hemostasis } \\
\text { Evidence-based Clinical Practice } \\
\text { Guidelines }\end{array}$ & 2014 & J Korean Med Sci 2014;29(2):164-171 & 70 \\
\hline $\begin{array}{l}\text { Asia-Pacific Thrombosis Advisory } \\
\text { Board consensus paper on prevention } \\
\text { of venous thromboembolism after } \\
\text { major orthopaedic surgery }\end{array}$ & 2010 & Thromb Haemost 2010;104(5):919-930 & 71 \\
\hline $\begin{array}{l}\text { Guidelines for the diagnosis, treat- } \\
\text { ment and prevention of pulmonary } \\
\text { thromboembolism and deep vein } \\
\text { thrombosis (JCS 2009) }\end{array}$ & 2011 & Circ J 2011;75(5):1258-1281 & 72 \\
\hline $\begin{array}{l}\text { Management of venous } \\
\text { thromboembolism }\end{array}$ & 2007 & J Assoc Physicians India 2007;55:49-70 & 73 \\
\hline $\begin{array}{l}\text { Malaysian Clinical Practice Guidelines: } \\
\text { prevention and treatment of venous } \\
\text { thromboembolism }\end{array}$ & 2013 & http://www.moh.gov.my/penerbitan/CPG2017/9005.pdf & 74 \\
\hline $\begin{array}{l}\text { The HKSCCM Position Statement: } \\
\text { Prevention of Venous Thromboem- } \\
\text { bolism in Intensive Care Units in } \\
\text { Hong Kong }\end{array}$ & 2010 & http://hksccm.org & 75 \\
\hline
\end{tabular}


Philippines, and South Asia including India, Pakistan, Afghanistan, Bangladesh and Sri Lanka.

A systematic search of the literature using the PICOT (Population, Intervention, Comparison, Outcome, Type) approach ${ }^{5}$ and the PRISMA checklist ${ }^{6}$ was conducted to identify English-language publications. The predetermined inclusion criteria were the following: (1) the population studied included at least $90 \%$ Asian participants or the study was conducted in one of the specified Asian countries; (2) the full publication was available in English; (3) the reported study included at least 100 subjects from the Asian population of interest; (4) the publication reported the disease burden and/or risk and demographic factors associated with symptomatic VTE; (5) the publication date was from 1995 to 2016.

\section{Data Collection and Analysis}

\section{Selection of Studies}

A wide, all-encompassing search string was developed through an iterative process and the final literature search was conducted in PubMed (MEDLINE) on 23 February 2016 (see Appendix A for search string). Two reviewers independently screened the title and abstract for each manuscript against the predefined inclusion and exclusion criteria (see - Fig. 1). Full-text manuscripts were obtained for all abstracts that were deemed potentially eligible by at least one reviewer. Two reviewers then independently screened the full-text articles for eligibility using a standardised data extraction form. Any discrepancies were resolved by discussion and/or consultation with a third reviewer $(n=42)$.

\section{Searching Other Resources}

The reference lists of all papers included were hand searched to identify any additional manuscripts that may have been omitted in the initial search; the 'related article' feature in PubMed was also used to identify additional articles. Google Scholar, EMBASE and the grey literature were also searched to cross-check for additional relevant articles (see $\boldsymbol{-}$ Fig. $\mathbf{1}$ ).

\section{Grouping of Studies}

Included studies were grouped, according to the study protocol, as belonging to one of the following categories: (1) populationbased studies, defined as population-based estimates of VTE incidence or mortality in the general population, from sources such as national health system registries or health insurance databases; (2) hospital evidence, defined as single institution or multicentre estimates of VTE incidence or mortality in the general population, or reports of VTE rates in consecutive hospitalized patients, surgical patients or general oncology patients; (3) autopsy evidence, defined as studies of PE incidence in series of autopsy records or death certificates; (4) special interest studies based on large population or patient groups; (5) studies of VTE in subpopulations or patient cohorts. Only high-quality studies with a low risk of bias were reported.

For population-based studies, ICD-9 or ICD-10-CM classifications were used. This system is the ninth or tenth revision of the World Health Organization International
Statistical Classification of Diseases and Related Health Problems (ICD) clinical modification (CM).

\section{Results}

The search strategy identified a total of 1,095 manuscripts. Initial title and abstract searches identified 227 potentially relevant studies. Hand searching of reference lists and additional searches identified a further 25 potentially relevant studies. Detailed abstract and/or full-text review identified 95 potentially relevant studies that met the criteria for data extraction. The following full-text review publications were excluded for the following reasons: small sample size (Supplementary references, 1-3); letter to the editor or literature review without new data (S4-S6); lack of denominator value, therefore no incidence reported or calculable (S7-S9); survey-based studies with poor response rates (S10-S13); duplicated data (S14); only non-symptomatic/sub-clinical disease, for example, VTE diagnosed by systematic screening with venous ultrasound imaging, or after D-dimer assay; incidence estimate extrapolated from less than $90 \%$ of the population of interest (S15); the report concerned predisposition for PE in people with a DVT(S16); or VTE was not a main study outcome of interest (S17). A total of 73 manuscripts met the eligibility criteria for data extraction (-Fig. 1). These were from Taiwan, Hong Kong, Singapore, China, Korea, Malaysia, Philippines, Thailand, Japan and India.

\section{Population-Based Estimates of Incidence}

Population-Wide Annual Incidences of VTE

Population-wide estimates derived from international classification of disease-coded population health data sets (ICD9 or ICD-10-CM) were identified from Korea, ${ }^{7}$ Taiwan $^{8}$ and Hong Kong ${ }^{9}$ (- Table 2).

The Korean study utilized a data set containing all inpatient and outpatient encounters recorded from 2004 to 2008 and investigated DVT alone and PE, with or without DVT. ${ }^{7}$ The Taiwanese study utilized data from the National Health Insurance programme, covering $99 \%$ of the population and $97 \%$ of hospitals and clinics, and recorded VTE rates from 2001 to 2002 in people older than 18 years. ${ }^{8}$ The Hong Kong study was derived from public hospital admission and discharge statistics in 2000 and 2001, covering 95\% of the population. ${ }^{9}$

In Korea, the age- and sex-adjusted annual VTE rates per 100,000 were 8.83 in 2004 and 13.8 in 2008. In Taiwan, the crude incidence of VTE was 15.9 per 100,000 person-years; in Hong Kong, the crude rates were 17.1 per 100,000 for DVT, 3.9 for PE and 19.9 for VTE (-Table 2).

A Japanese study reported death certificate data in which death was attributed to PE, as recorded in 'Vital Statistics of Japan' from 1951 to 2000, and data were adjusted to the population size in 1985 . The reported rate of 'fatal PE' increased from 0.08 per 100,000 in 1956 to 0.77 per 100,000 in $1996 .^{10}$

\section{Population-Based VTE Rates in Patients with Specific Chronic or Acute Disorders}

We identified 24 population-based retrospective cohort studies of specific disorders, injuries, toxicities or drug therapies, 


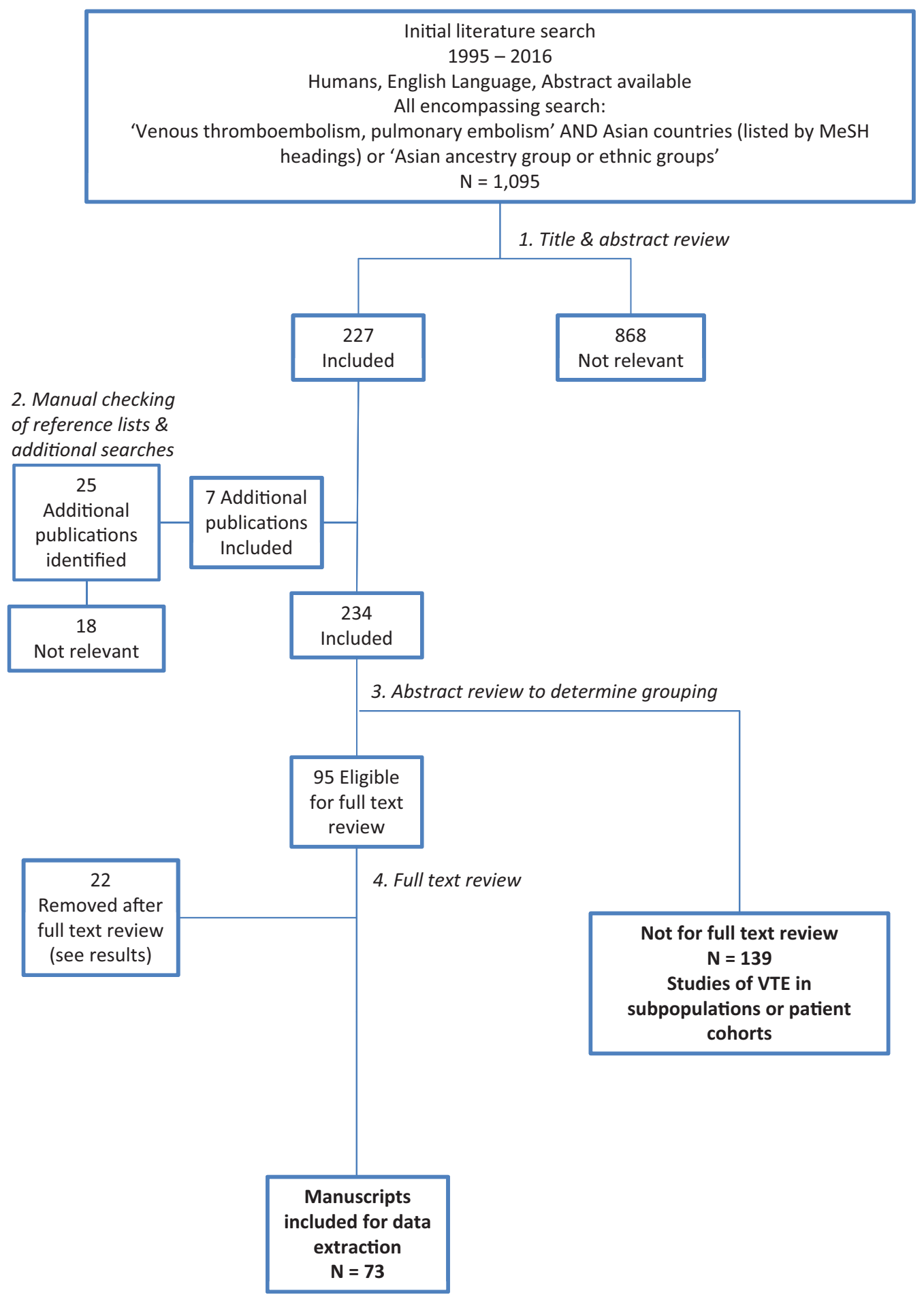

Fig. 1 Flow chart.

all derived from the Taiwan National Health Insurance databases. Each study reported absolute DVT, PE or VTE rates in cases and matched controls, together with adjusted incidence hazard ratios (HR; - Table $\mathbf{3}$ ).

\section{Medical Disorders, Injuries and Toxicities}

The estimated incidence of PE was significantly higher in patients with chronic inflammatory disorders than in ageand sex-matched controls. The disorders included systemic lupus erythematosus (SLE; PE rate of 10.2 per 10,000 patientyears; HR: 19.7; 95\% confidence interval [CI]: 11.9; 32.8), ${ }^{11}$ systemic sclerosis (SSc) (10.8 per 10,000 patient-years), ${ }^{12}$ chronic obstructive pulmonary disease (COPD; 12.31 per 10,000 patient-years) ${ }^{13}$ and asthma (10.2 per 10,000 patientyears) ${ }^{14}$ Estimated incidence rates of DVTwere also significantly higher in patients with COPD, ${ }^{13,15}$ SLE, ${ }^{11}$ Sjogren's syndrome (SS), ${ }^{16}$ SSc, ${ }^{12}$ rheumatoid arthritis, ${ }^{17,18}$ inflammatory bowel disease (IBD), pneumococcal pneumonia, ${ }^{19}$ osteomyelitis, ${ }^{20}$ 


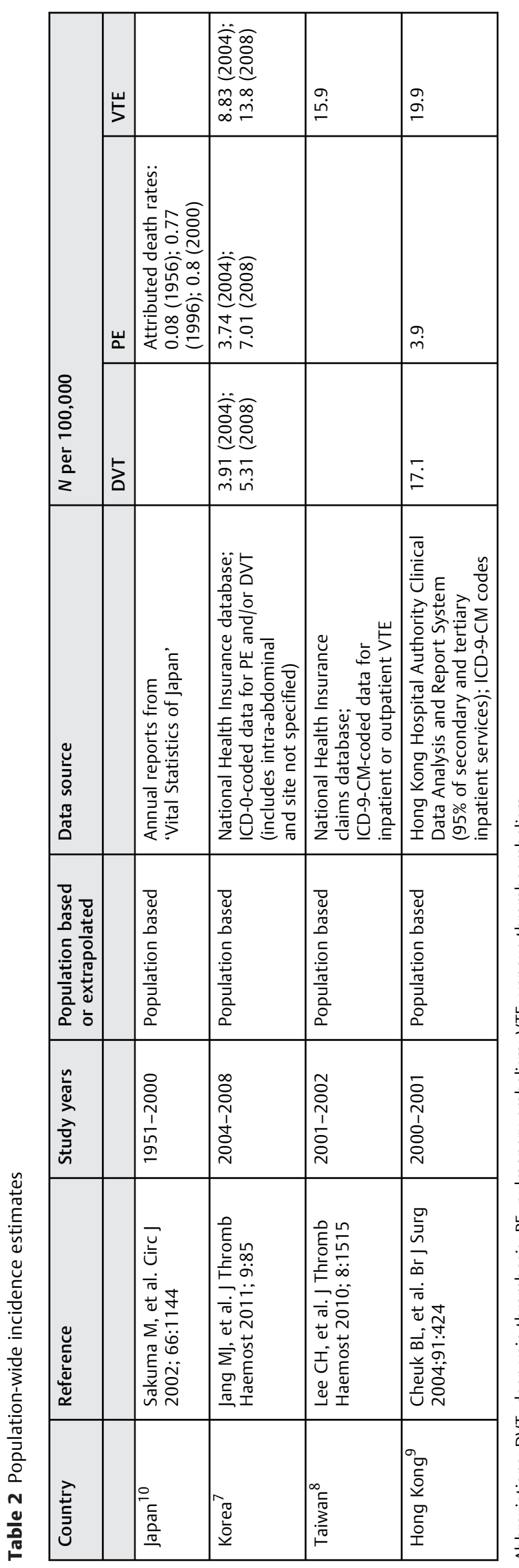

tuberculosis ${ }^{21}$ and hepatitis $\mathrm{C}$ virus infection ${ }^{22}$ compared with controls (-Table 3). Two studies only recorded VTE incidence, and both showed significantly higher rates in patients with dermatomyositis (HR: 11.1; 95\% CI: 5.2, 23.6) and liver cirrhosis (HR: 7.7; 95\% CI: 5.2, 11.4) 23 $^{23}$ compared with controls.

Other conditions with higher estimated incidence rates of DVT or VTE, compared with controls, included spinal cord injury, ${ }^{24}$ organophosphate toxicity, ${ }^{25}$ carbon monoxide poisoning, ${ }^{26}$ schizophrenia, ${ }^{27}$ sleep disorders, ${ }^{28}$ hormone replacement therapy ${ }^{29}$ and type 2 diabetes mellitus. ${ }^{30}$ Metformin use in type 2 diabetes was associated with a reduced risk of DVT with $0.22 \%$ of cases compared with $0.56 \%$ of controls affected (HR: 0.43 ; $95 \%$ CI: $0.24,0.56 ;{ }^{31}$-Table 3).

\section{Post-surgical VTE Rates}

Seven population-based studies reported the rates of VTE recorded within 4 to 5 weeks, or within 3 months, of surgery ${ }^{32-38}$ (-Table 4). Five studies were of patients who had major joint surgery (Taiwan $n=3$; Korea $n=2$ ), another (Korea) examined VTE rates after major surgery (including joint surgery) and a study from Japan investigated rates after spinal surgery. The estimated incidences of symptomatic VTE following total hip arthroplasty (THA) were $0.15,{ }^{37} 0.27,{ }^{32} 0.40,{ }^{34} 0.98^{36}$ and $1.35 \%$ 39 in five separate studies. After total knee arthroplasty (TKA), the reported incidence ranged from 0.22 to $1.2 \%$. Hip fracture surgery $(1.60 \%)$ and cancer surgery $(0.67 \%)$ were associated with high VTE incidence rates in Korea $^{36}$ ( - Table 4).

\section{Pregnancy-Related VTE Rates}

Only one large-scale population study investigating pregnancyrelated VTE rates was identified, and reported the annual rate increased from 2006 to 2010 in South Korea (the overall incidence was 0.82 per 10,000 deliveries during the 5 -year period). ${ }^{40}$

\section{VTE as a Hospital Discharge Diagnosis}

Prevalence of VTE among All Hospital Inpatients

Nine studies ${ }^{9,41-48}$ reported the number of patients with a discharge diagnosis of DVT, PE or VTE per 10,000 hospital inpatients, as recorded in hospital or registry databases in mainland China, Hong Kong, India, Japan, Korea and Singapore. These ranged widely from 11 to 65 per 10,000 for DVT, 2.5 to 23 per 10,000 for PE and 11 to 88 per 10,000 for VTE. In Singapore, the prevalence of DVT among inpatients increased from 16 cases per 10,000 admissions in 1996/1997 to 65 cases per 10,000 admissions in 2006 (-Table 5).

\section{Prevalence of VTE in Medical Inpatients}

Three studies of symptomatic VTE among medical or 'high-risk' medical inpatients in Thailand and Singapore ${ }^{49}$ and one study of VTE after an ischaemic or haemorrhagic stroke in Taiwan are shown in - Table 6. The reported VTE prevalence was 0.2 to $0.9 \%$ of inpatients ( $1.6 \%$ in patients with chronic liver disease).

\section{Prevalence of VTE among Surgical Inpatients}

The rates of symptomatic post-surgical VTE in 10 single hospital studies or multicentre registries from East Asia, 


\begin{tabular}{|c|c|c|c|c|c|c|c|c|c|c|c|c|c|c|c|}
\hline \multirow{3}{*}{ 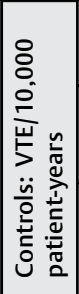 } & $\breve{5}$ & & & & & & & & & 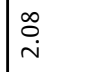 & & & $\stackrel{\stackrel{n}{m}}{m}$ & & \\
\hline & \multirow{2}{*}{$\begin{array}{l}\text { uㅗ } \\
5 \\
5\end{array}$} & f. & 官 & 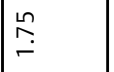 & & $\stackrel{\text { 무 }}{\longrightarrow}$ & $\frac{\stackrel{n}{\sim}}{i}$ & $\frac{\varphi}{m}$ & $\begin{array}{l}g \\
\dot{m}\end{array}$ & & \begin{tabular}{l}
\multirow{2}{*}{} \\
0
\end{tabular} & & & 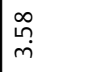 & 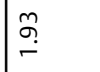 \\
\hline & & $\stackrel{m}{\rightleftarrows}$ & $\underset{m}{\bar{m}}$ & $\underset{\sim}{\tilde{m}}$ & & $\stackrel{\infty}{\stackrel{\infty}{\rightarrow}}$ & $\stackrel{\stackrel{n}{+}}{\stackrel{n}{*}}$ & & & & & $\begin{array}{l}\stackrel{\rho}{\sim} \\
\ddot{r}\end{array}$ & $\stackrel{\nabla}{\sigma}$ & $\mid \begin{array}{r}\hat{R} \\
6\end{array}$ & $\begin{array}{l}\bar{n} \\
m\end{array}$ \\
\hline 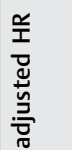 & $\overrightarrow{5}$ & & & & & & & & & 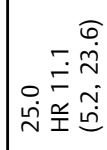 & & & & & \\
\hline 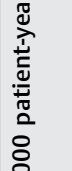 & шّ & 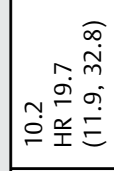 & 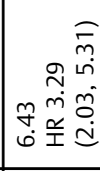 & 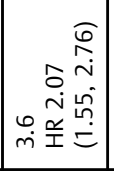 & & 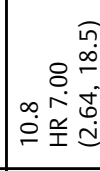 & 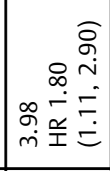 & 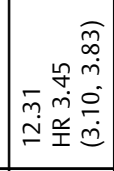 & 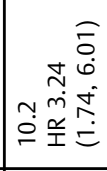 & & 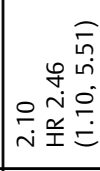 & & 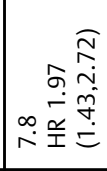 & 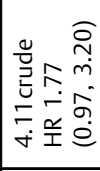 & 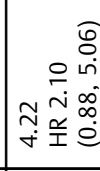 \\
\hline 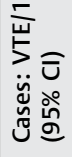 & 5 & 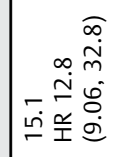 & 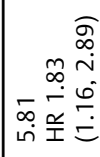 & 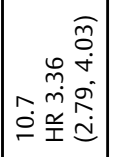 & 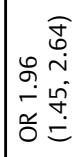 & 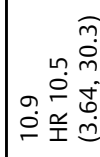 & 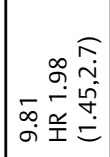 & & & & & 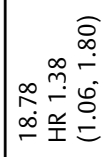 & 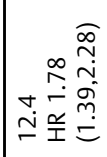 & 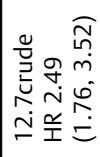 & 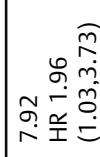 \\
\hline 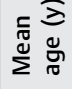 & & 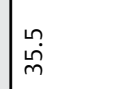 & 吕 & ก & & in & กิ & 反 & m & $\mid \begin{array}{l}\stackrel{n}{\sim} \\
\dot{\sigma}\end{array}$ & 哃 & $\approx$ & 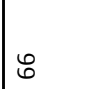 & 㤐 & $\frac{9}{\dot{n}}$ \\
\hline 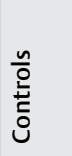 & & 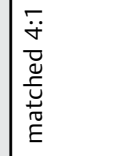 & 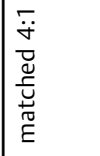 & 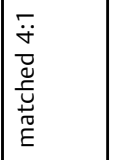 & 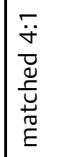 & 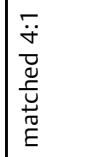 & 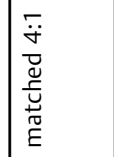 & 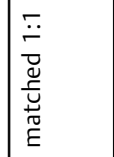 & 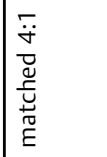 & 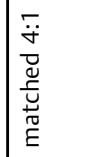 & 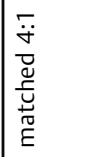 & 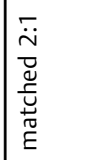 & 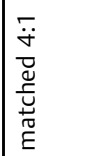 & 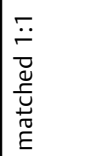 & 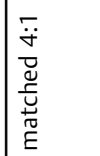 \\
\hline 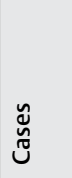 & & 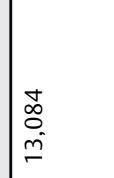 & $\begin{array}{l}\text { స్ } \\
\text { - }\end{array}$ & $\begin{array}{l}\infty \\
\tilde{N} \\
\tilde{N} \\
\tilde{N}\end{array}$ & 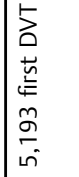 & $\begin{array}{l}\text { n. } \\
\infty \\
\end{array}$ & 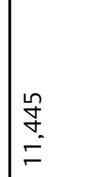 & 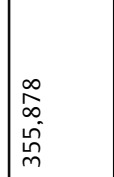 & \begin{tabular}{|l}
$\stackrel{L}{L}$ \\
$m$ \\
$m$ \\
$m$ \\
$m$
\end{tabular} & $\begin{array}{l}\overline{\tilde{m}} \\
\hat{i}\end{array}$ & $\begin{array}{l}\infty \\
\infty \\
o \\
\sigma\end{array}$ & 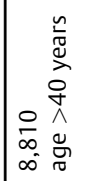 & 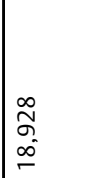 & 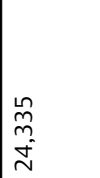 & $\mid \begin{array}{l}0 \\
0 \\
0 \\
m \\
\text { r. }\end{array}$ \\
\hline 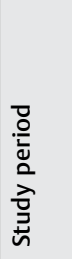 & & 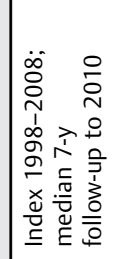 & 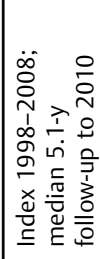 & 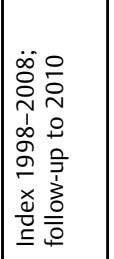 & 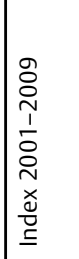 & 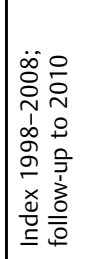 & 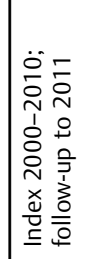 & 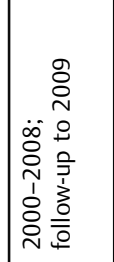 & 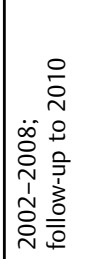 & 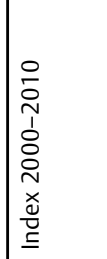 & 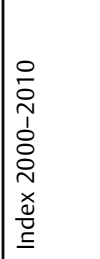 & 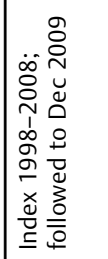 & $\begin{array}{l}0 \\
0 \\
0 \\
1 \\
1 \\
\infty \\
2 \\
1 \\
1\end{array}$ & 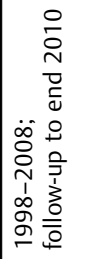 & 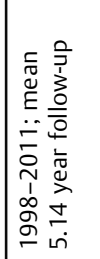 \\
\hline 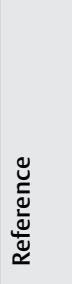 & & 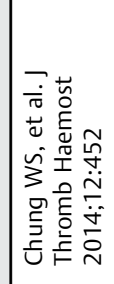 & 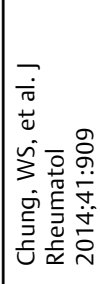 & 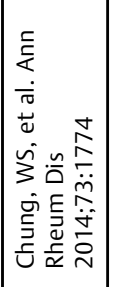 & 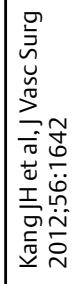 & 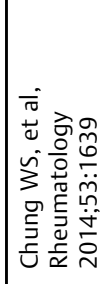 & 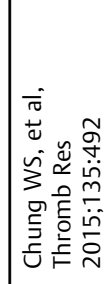 & 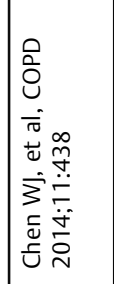 & 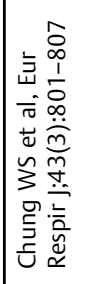 & 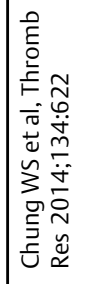 & 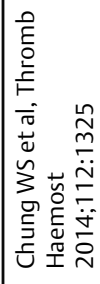 & 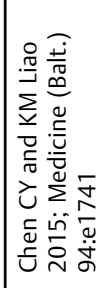 & 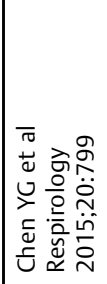 & 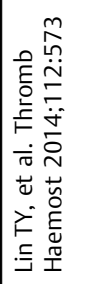 & 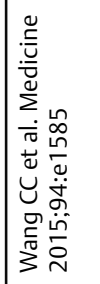 \\
\hline 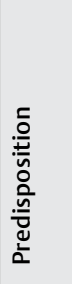 & (5) & 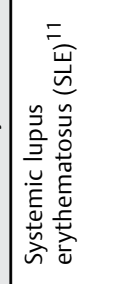 & 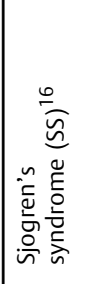 & 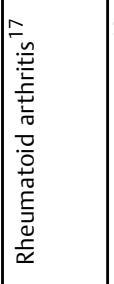 & 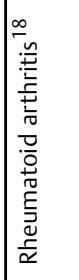 & 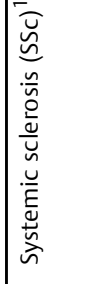 & 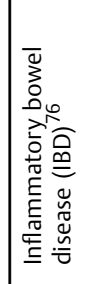 & 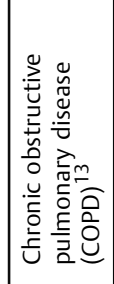 & 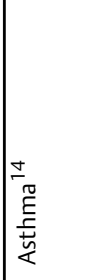 & 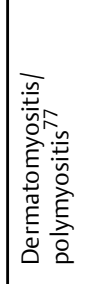 & 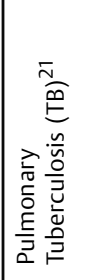 & 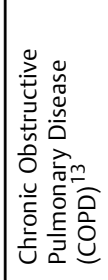 & 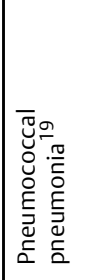 & 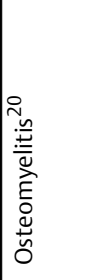 & 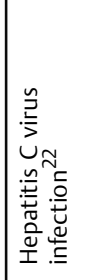 \\
\hline
\end{tabular}




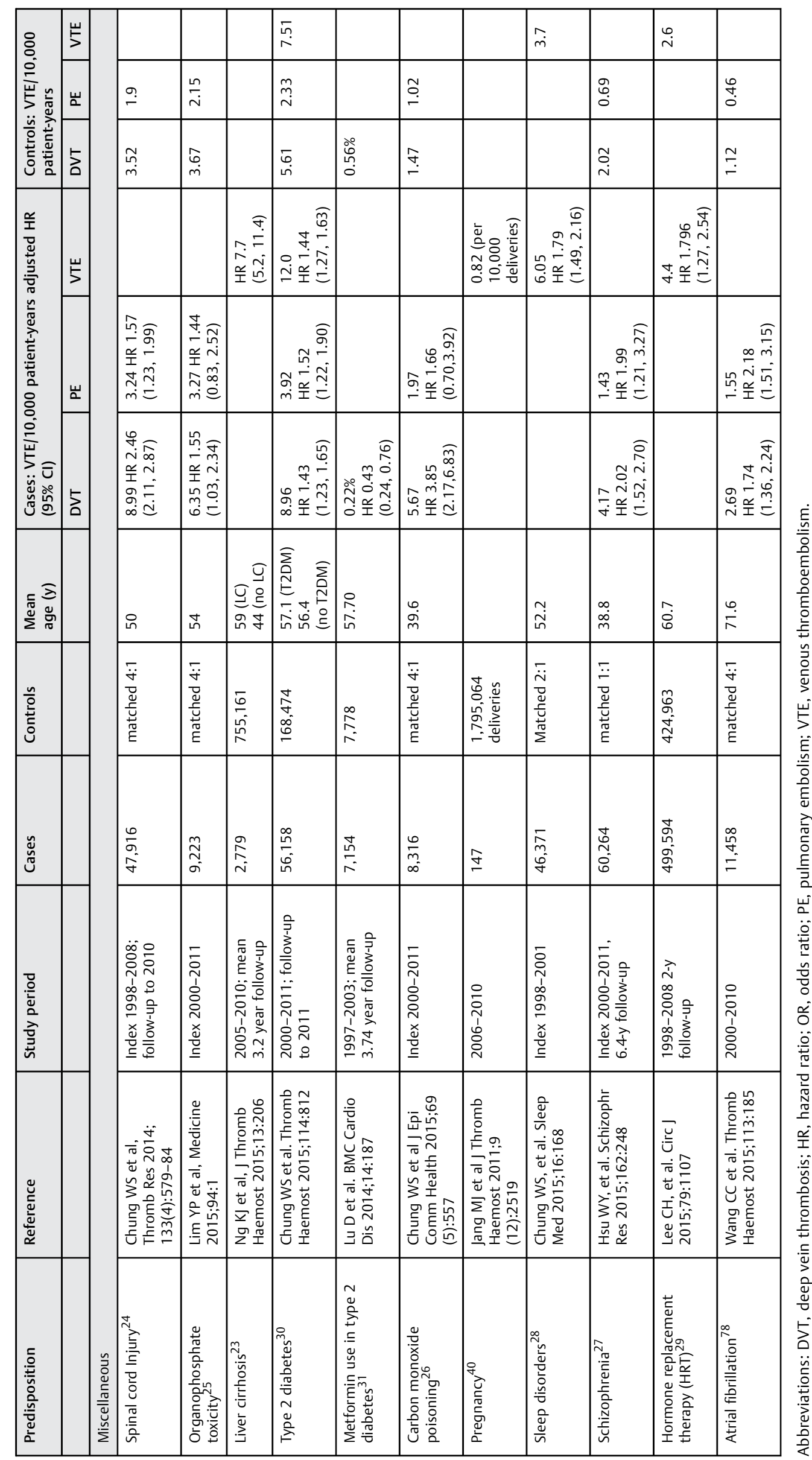




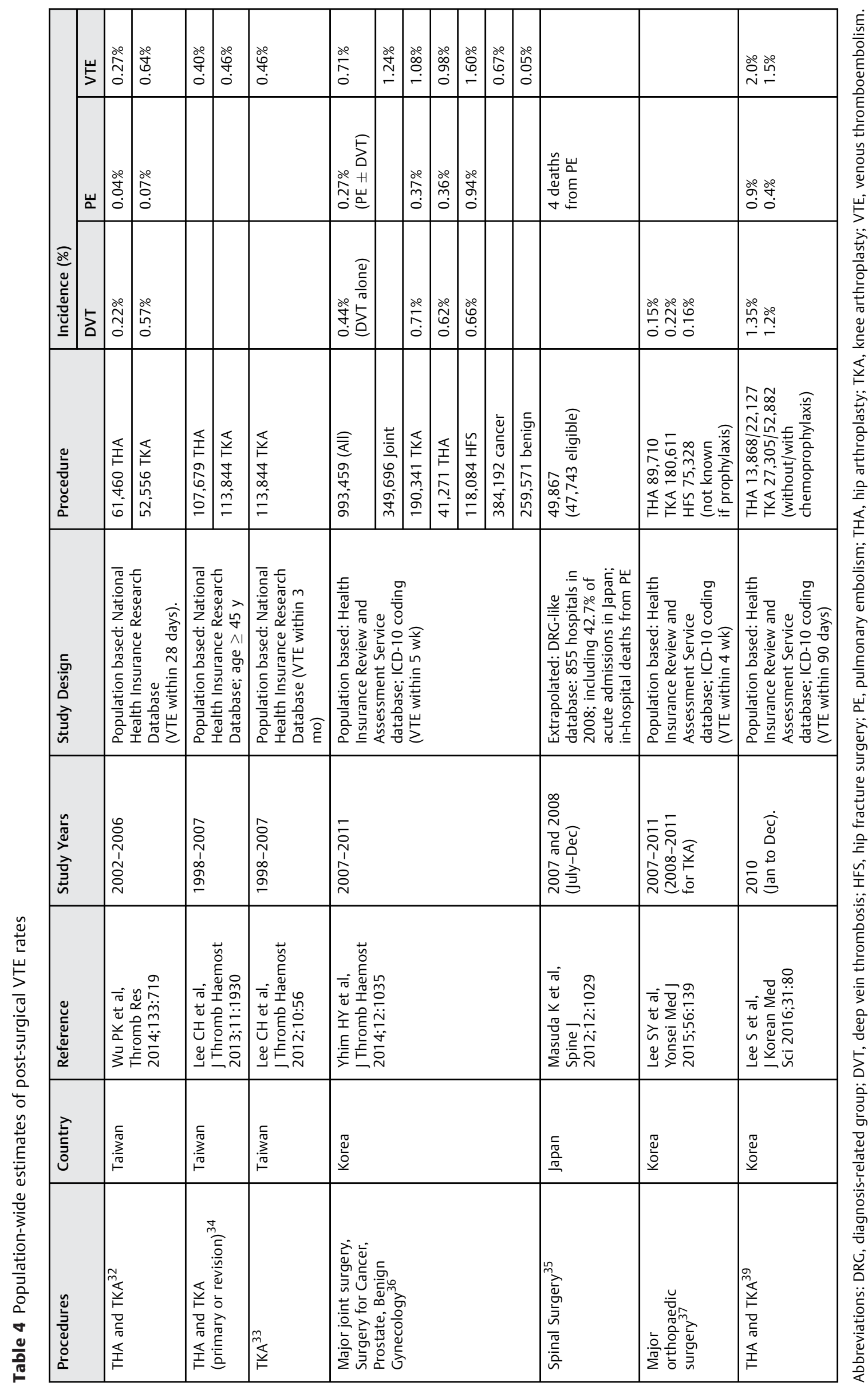




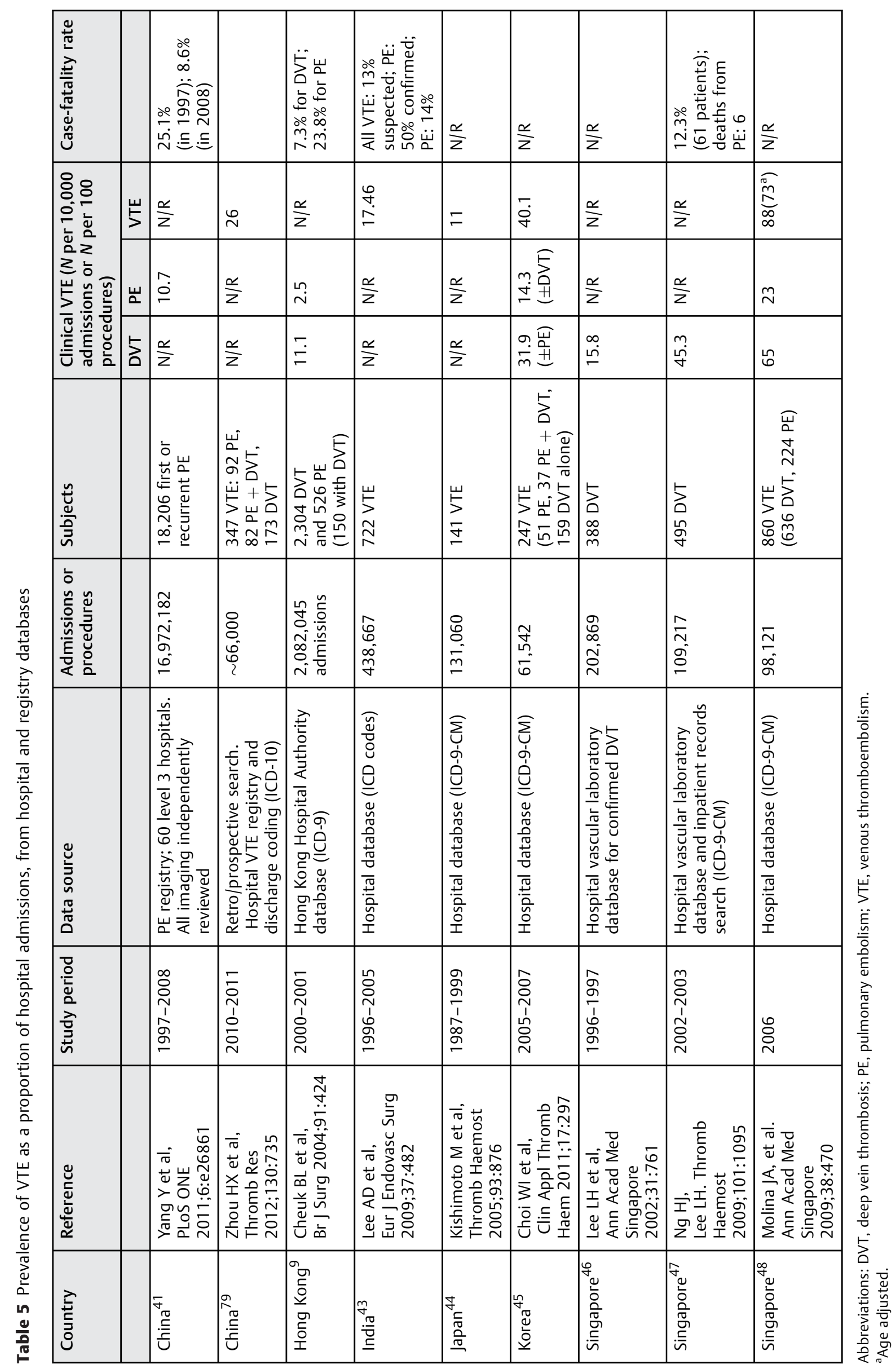




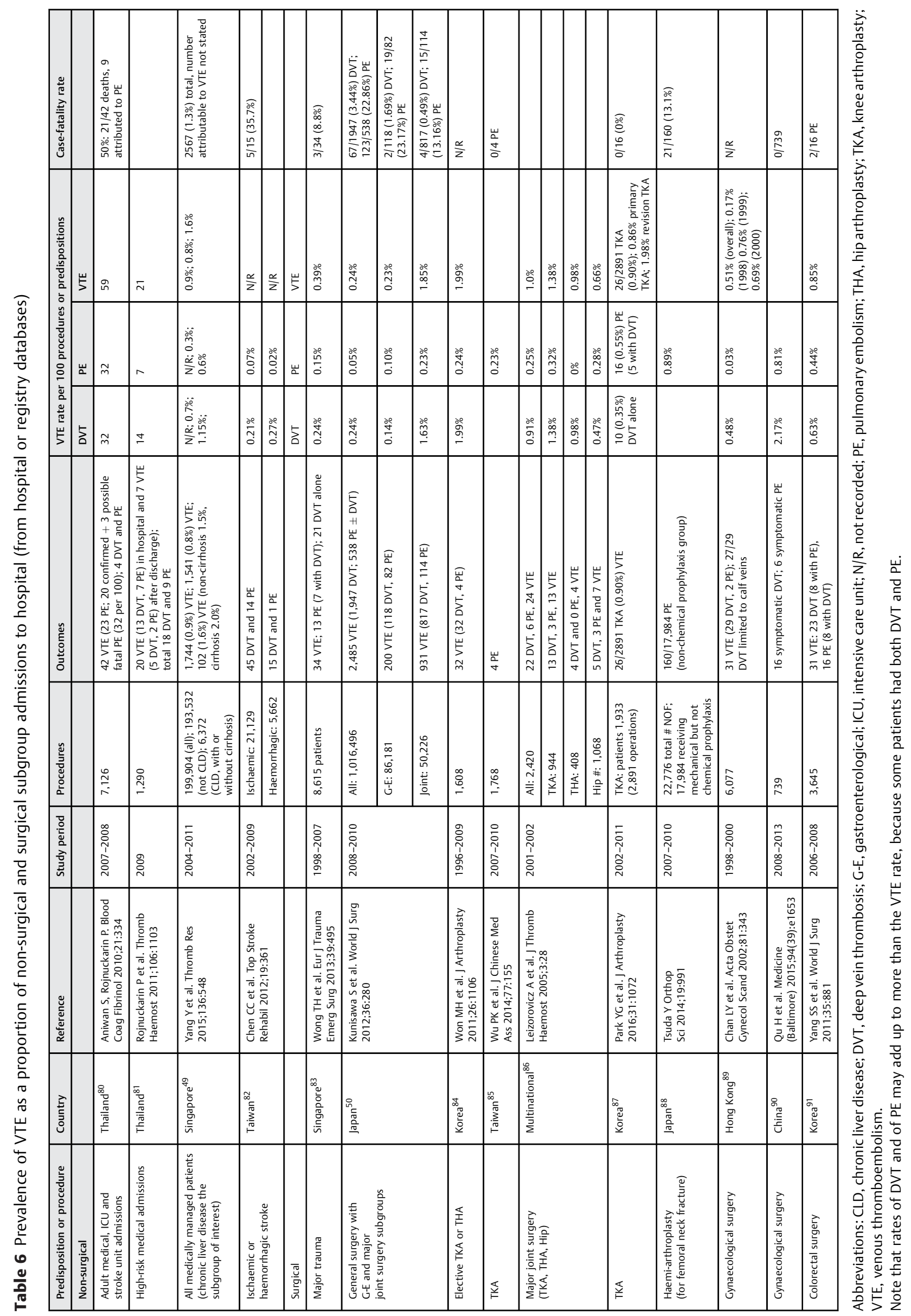


with various times of clinical follow-up, are presented in - Table 6. The reported rates were $0.39 \%$ (major trauma), 0.23 to $0.24 \%$ (general and gastric and bowel surgery), $0.85 \%$ (colorectal surgery), $0.51 \%$ (gynaecological surgery in Hong Kong [0.17\% in 1998, rising to $0.76 \%$ and $0.69 \%$ in 1999 and 2000]; higher in mainland China), $0.66 \%$ after hip fracture and 1.0 to $1.85 \%$ after major joint arthroplasties.

\section{Old Age, Gender and Other Potentially Contributing Conditions}

\section{Old Age}

Four population-based studies ( - Table 2 ) describe strong associations of VTE risk with advancing age. In Korea, relative to the 30- to 39-year age group, there was a sixfold increase in risk by age 60 to 69 years, and a 15 -fold increase by age greater than 80 years when the absolute annual rate was just over $1 / 1,000$ people. ${ }^{7}$ The absolute annual VTE rate of 0.02 per 1,000 women and 0.03 per 1,000 men aged less than 30 years, increased to 1.2 and 0.8 per 1,000 among women and men aged greater than 80 years. ${ }^{8}$ Observations were similar in Hong Kong and Japan. ${ }^{9,10}$

\section{Gender}

Three population-wide studies reported on gender and VTE risks. In one study, women were less likely to have a diagnosis of VTE (relative risk [RR]: 0.96) or DVT alone (RR: 0.87) but more likely to have a diagnosis of PE (RR: 1.11). ${ }^{7}$ In a second study, $54 \%$ of patients with VTE were female, ${ }^{8}$ while the third study reported no significant difference. ${ }^{9}$ Women slightly outnumbered men in seven of nine hospital or registry-based cohorts of patients with VTE (a tendency most pronounced in two of the three studies from Japan; - Table 7).44,50

\section{Other Comorbidities and Potential Predispositions}

In Taiwan, a population-based analysis of VTE rates and associated risk factors, or predispositions, showed that $16 \%$ of cases had previous VTE, and most VTE was associated with predisposing conditions (22\% malignancy, $17 \%$ serious neurological disease, $8 \%$ major trauma $8 \%$ and $38 \%$ major surgery during the preceding 3 months), although $27 \%$ had no recorded predisposition. ${ }^{8}$ Similar potentially contributing conditions were recorded among hospital inpatients with VTE (-Table 8): age older than 70,75 or 80 years (15-42\% of patients); cancer $(13-52 \%$ of patients); previous VTE (1-16\% of patients); immobility (up to $74 \%$ of patients), acute or chronic heart or lung conditions (up to $36 \%$ of patients); stroke or paresis; various inflammatory disorders; and a body mass index (BMI) $\geq 25 \mathrm{~kg} / \mathrm{m}^{2}$.

\section{Cancer-Related Venous Thromboembolism}

- Table 8 shows the incidences of symptomatic VTE in patients with various cancers, reported from East Asia. One populationbased cohort study from Taiwan searched all hospital admissions for any form of newly diagnosed cancer; ${ }^{51}$ another searched a randomly selected $15 \%$ subset of all admissions. ${ }^{52}$ The studies estimated that 1.85 and 3.4 patients per 1,000 patient-years of follow-up developed VTE (using somewhat different identifying algorithms). VTE was more likely if there was previous VTE, ${ }^{51}$ or cancer of the pancreas, lung or liver, a sarcoma or multiple myeloma, ${ }^{52}$ and VTE was associated with reduced survival. In a large cohort of Korean patients with non-small cell lung cancer (NSCLC), 6.4\% developed VTE during 2 years of observation, and this was associated with reduced survival if patients had limited stage disease..$^{53}$ In a study from China, $1.3 \%$ of patients with any form of lung cancer developed VTE (all VTE patients had NSCLC). ${ }^{54}$ Patients with a gynaecological malignancy had VTE rates of $3.3 \%$ during 5 years of follow-up in a population-based study from Taiwan, ${ }^{55}$ and $1.3 \%$ in a hospital cohort from Thailand (where most VTE was associated with ovarian cancer) ${ }^{56}$ The 2 -year incidence of VTE was $4.9 \%$ after inoperable gastric cancer (metastatic or recurrent) in a cohort from Korea. ${ }^{57}$ VTE rates were $1.4 \%$ during 4 months after starting thalidomide for relapsed or refractory multiple myeloma in Japan, ${ }^{58}$ and less than $1 \%$ in Korean patients with acute myeloid leukaemia. ${ }^{59}$

\section{Other Observations: Recurrence Rates and Case Fatality Rates}

A population-wide study of over 5,000 Taiwanese adults discharged from hospital after an admission with VTE in 2001 or 2002 found that annual crude recurrence rates were $5.1 \%$ in men and $5.2 \%$ in women, during up to 4 years (average 2.26 years) of follow-up. ${ }^{8}$ A recurrence was most likely during the first 12 months after VTE (with cumulative rates of 4.6, 6.7 and $9.4 \%$, after 3, 6 and 12 months, respectively, which reached $14.4 \%$ at 4 years), and was three to four times more likely in patients with a previous VTE or cancer than if the predisposition was transient or the index VTE was apparently unprovoked. ${ }^{8}$

This and another population-based report found high case fatality rates. In Taiwan, $4.3 \%$ of patients with VTE died in hospital, 7.5\% died within 1 month and 14.9\% died within 6 months; mortality was higher after PE than DVT. ${ }^{8}$ In Hong Kong, $7.3 \%$ of admissions with a DVT and $23.8 \%$ of those with PE died in hospital, and VTE-associated mortality was greatest in the elderly. ${ }^{9}$ High 30 -day or in-hospital mortalities were also reported in a questionnaire-based survey from Japan (7\% after DVT, $13 \%$ after PE), ${ }^{60}$ a PE registry from China (9\% in 2008) ${ }^{41}$ and hospital cohorts in Singapore $(12 \%)^{47}$ and India (13\%). ${ }^{43}$

\section{Temporal Trends}

The reported incidence of VTE has increased during recent decades, in population-wide studies from Korea and Japan (see above) in registries, and in hospital databases. There was an almost fivefold increase in the annual number of patients recorded between 1997 and 2008 in a very large Chinese PE registry, ${ }^{41}$ and a fivefold rise in admissions with acute DVT at a Singaporean hospital between 1990 and $1997 .^{46,47}$ In China, the start of a nationwide project to improve diagnosis and management of VTE coincided with a sharp upturn in the number of patients registered as having PE. ${ }^{41}$

\section{Discussion}

This systematic literature review of evidence regarding the population-based incidence of symptomatic VTE in East Asian populations confirms the frequency and importance of this 


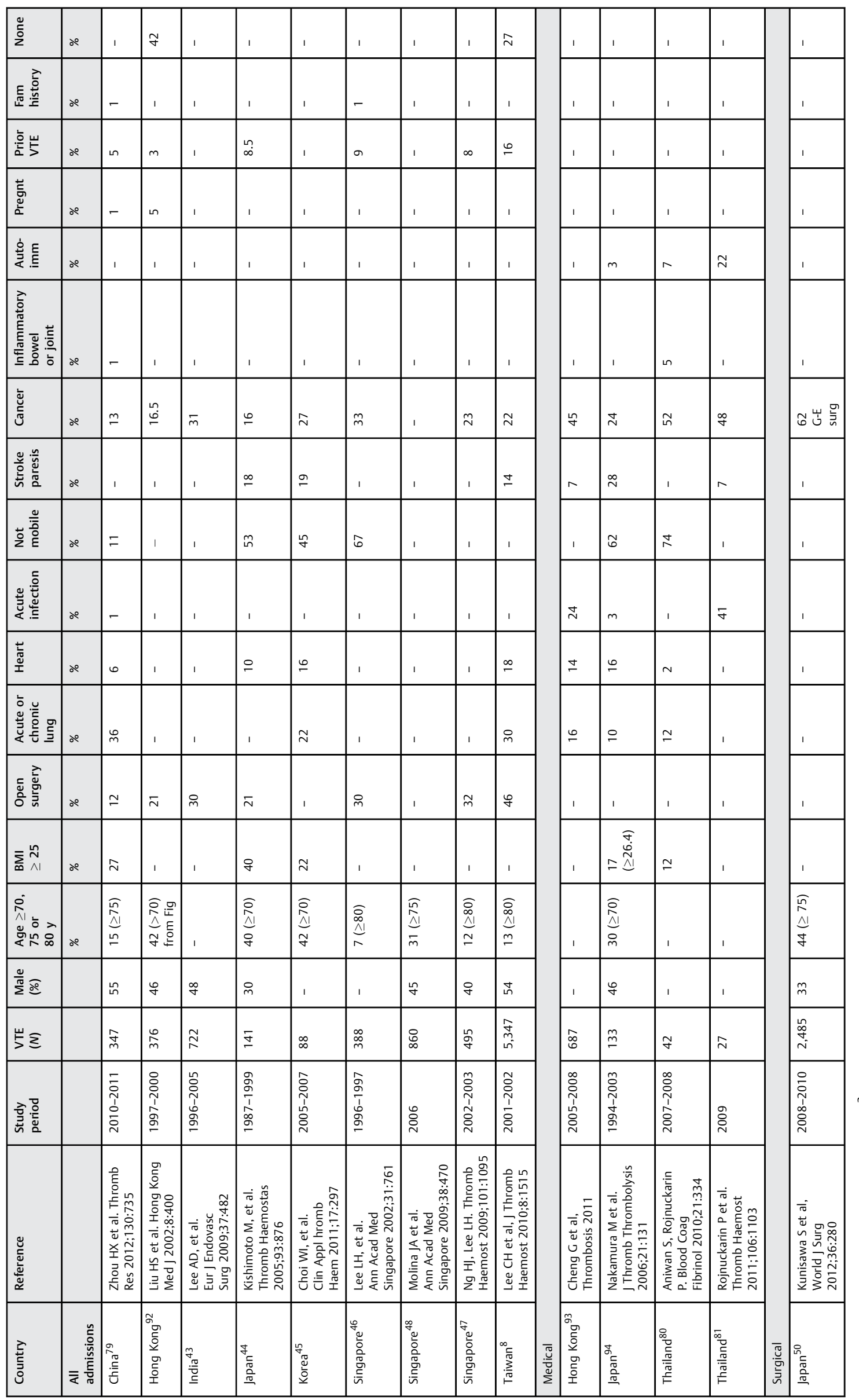




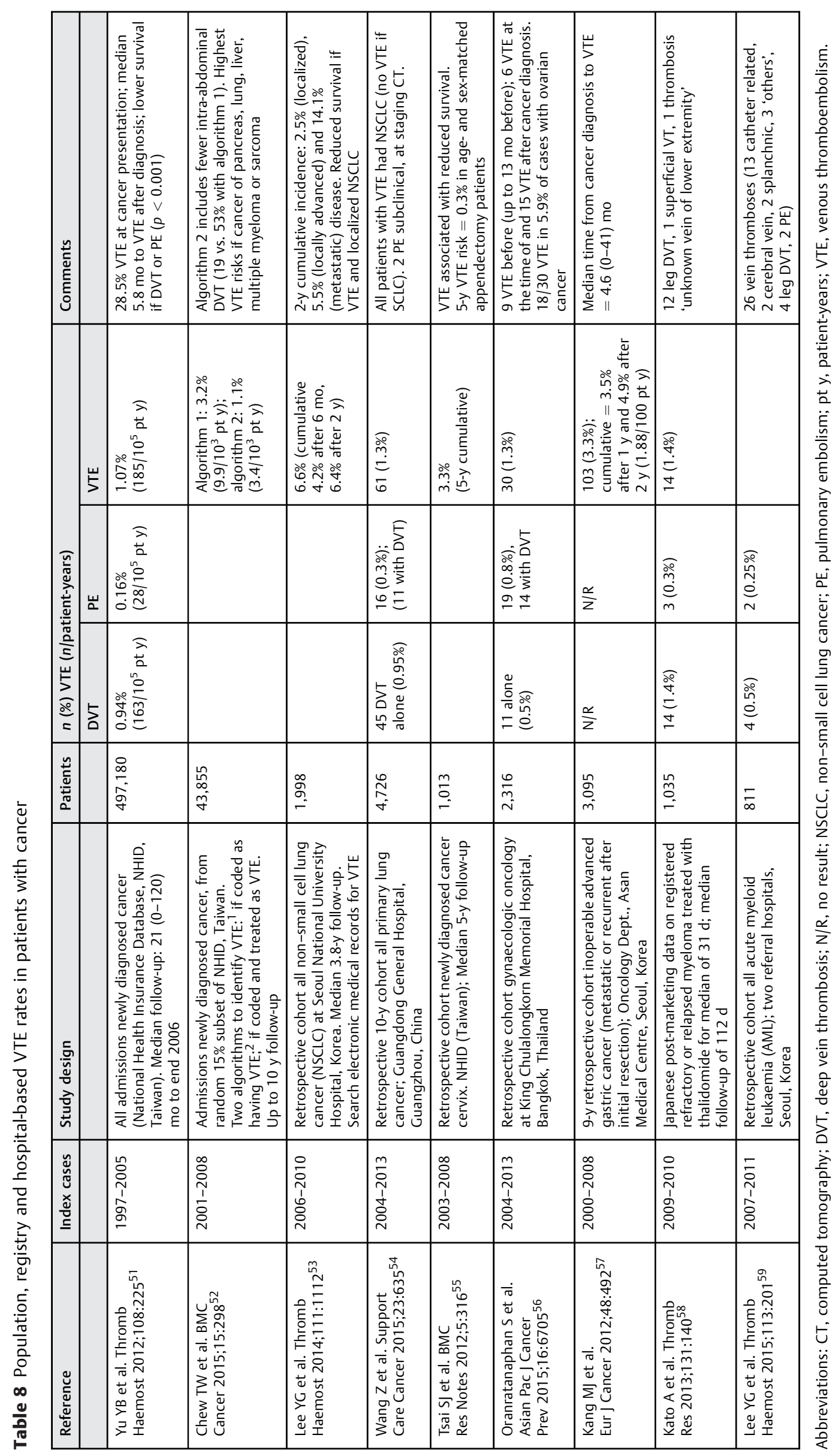


disease is increasing, especially among the aged. Our search for evidence was focused on symptomatic VTE, because it is symptomatic DVT or PE that determines the clinical burden from VTE.

\section{VTE Incidence in Asia Compared with the West}

Recently published population-wide estimates of annual symptomatic VTE rates in Korea, Taiwan and Hong Kong are approximately 15 to $20 \%$ of the level recorded in Western countries ( $\sim 100$ per 100,000 people). ${ }^{61}$ However, the reported annual incidence in East Asian populations increases to approximately 1 per 1,000 people aged $\geq 80$ years, ${ }^{7-9}$ as compared with 3 per 1,000 in some Caucasian populations. ${ }^{57}$ In addition, the National Health Insurance data from Korea, Japanese vital statistics of deaths attributed to PE, data from PE registries and hospital admission statistics all indicate a rise in reported VTE rates over time across East Asia. ${ }^{7,8,46}$

Hospital data from large registries and databases corroborate the findings in population-health studies and potentially provide more accurate and robust estimates of VTE rates in certain patient groups (-Table $\mathbf{5}$ ). Across nine studies, the prevalence of VTE and the increasing temporal trend across three studies from Singapore echoed that of population-based studies. Rates of symptomatic, post-operative VTE (- Table 6) are difficult to compare with Western values due to differing methodologies. In a South Korean population-based study, ${ }^{40}$ the incidence of pregnancy-related VTE was 0.82 per 10,000 deliveries, much lower than the Western rates of between 10 and 20 per 10,000 deliveries.

\section{Accuracy of Population-Based Datasets}

Estimates of population-wide VTE rates, based on populationwide databases, not only require these to include both inpatient and outpatient encounters, but also rely on accurate reporting using International Classification of Diseases (ICD) or local disease classification, evidence of anticoagulant therapy and the assumption that every patient at discharge or at censure had confirmatory tests or a post-mortem to identify VTE. Therefore, any attempt to estimate population-wide VTE rates has limitations and inherent biases. There is a risk of under-reporting, because VTE must be identified in the medical record to ensure a correct discharge diagnosis, and clinically significant PE or DVT is often misdiagnosed or unrecognized. ${ }^{62}$ It is also important to note that the algorithms used to define DVT, VTE and PE based on ICD-9 or ICD-10 codes can vary from study to study and result in inaccurate estimation. For example, in some studies, the category DVT also included inferior vena cava (IVC) thrombosis, renal vein thrombosis and/or thrombophlebitis. ${ }^{7-9}$

A systematic review of studies done to validate the identification of VTE using administrative and claims data showed that reliability varies considerably and positive predictive values (PPVs) are highest when multiple codes are combined. ${ }^{63}$ The range of PPVs reported using combined ICD-9 codes for DVT, PE or VTE was 65 to $95 \%$. Ng et al ${ }^{23}$ also validated their Taiwanese database by medical chart review of a random sample and found a PPV of $94.0 \%$ for VTE.

The strengths of population-based data include large sample size and ability to capture the entire population.

\section{Risk Factors and Recurrence}

It is of interest that the comorbidities and likely predispositions for VTE are similar in East Asia and in the West, most notably old age, cancer, previous VTE, immobility, trauma and surgery, and inflammation. Of particular note are the population-based cohorts with case-control comparisons, from Taiwan, which show high HR for VTE in many acute or chronic autoimmune and other inflammatory disorders (e.g. systemic lupus, SS and rheumatoid arthritis) compared with controls. There is a noteworthy finding that DVT rates were less in type 2 diabetes patients prescribed metformin; ${ }^{31}$ recent laboratory studies have shown that metformin inhibits platelet activation and release of mitochondrial DNA (deoxyribonucleic acid). ${ }^{64}$

Apart from the South Korean population study in pregnancy-related VTE, ${ }^{40}$ it is important to acknowledge the lack of published evidence from large VTE studies in Asian populations for the predispositions of pregnancy, the perinatal and post-partum period, and gynaecological laparoscopic surgery.

The overall recurrence rate of $10 \%$ during the first year after presenting with VTE, recorded in a population-based study from Taiwan, is similar to 1-year recurrence rates reported in the West of 5.6 to $12.9 \% .{ }^{65}$ Surprisingly, the risks of a recurrence after a transiently provoked or supposedly unprovoked VTE were similar ( $\sim 5 \%$ during 1 year), which suggests that Western guidance on treatment duration should be validated in Asia.

\section{Lower Asian Venous Thromboembolism Rates}

There has been much speculation about why the reported VTE rates might be lower in Asia than the West. ${ }^{61,66}$ Explanations have included under-diagnosis, low clinician awareness and the near absence in Asia of thrombophilias commonly found in Caucasian populations, such as factor $\mathrm{V}$ Leiden and prothrombin gene mutations. Yet this review has confirmed a clear trend of increasing VTE rates over time, across multiple population- and hospital-based studies. It is likely that in part under-diagnosis and under-reporting in early studies, followed by improved diagnosis in later years with greater awareness and perception that VTE is an issue in Asian populations, have contributed to this trend. In Singapore ${ }^{47}$ and Hong Kong, ${ }^{67}$ it was shown that reported VTE rates have increased as Doppler ultrasound scans were used more often and the institutions established a lower threshold for performing diagnostic tests. Similarly, PE rates in China surged following a nationwide programme to improve awareness and management of this condition.

Nevertheless, it seems likely that there has also been a genuine increase in VTE prevalence in Asian populations, perhaps because of increasing population age, increasing cancer rates and an increasing number of surgeries. The rates reported in elderly people from Asian countries suggest that the gap between VTE rates in Asia and the West will diminish further still.

\section{Improving Awareness of VTE in Asia}

A recent survey found variable public awareness of VTE worldwide and very low awareness in Asia. ${ }^{66}$ Low and 
inadequate clinician awareness as well as low public awareness of VTE in Asia ${ }^{61}$ is likely to result in a general trend towards under-diagnosis. This is exemplified by the Chinese PE registry, where a sharp upturn in the number of reported patients with PE coincided with a nationwide programme to improve awareness and management of this condition. ${ }^{9}$ The increase in reported VTE rates over time recorded by several studies identified in this systematic review suggests initial under-diagnosis and rising diagnostic awareness. Most of the population-based studies reviewed here were based on data collected more than 10 years ago (2000-2008), and the current VTE rates may be considerably higher.

Implications for Guidelines and Other Asian Countries Our estimates of population burden from VTE in East Asia come from Hong Kong, Korea, Taiwan and (with less methodological confidence) Singapore. It is important to stress that these estimates should not be directly extrapolated to places with different health care systems and less extensive databases, so that similar statistics from other countries in the region such as India, Malaysia, the Philippines and Indonesia are much needed. Data on the population disease burden of VTE across Asia will also allow racial and ethnic differences in VTE to be better studied.

-Table 1 lists guidelines and expert recommendations from Asia about VTE risk assessment, prevention and management. Several of these include summaries of the literature on VTE rates and VTE prevention trials in Asia. Most base their recommendations for anticoagulant prophylaxis, explicitly or implicitly, on international guidelines derived from Western data for Western populations, where the balance of absolute benefit and absolute risk may not be the same as in Asia. This and other recent surveys of evidence ${ }^{68}$ find an increasing incidence of VTE in Asian populations. This emphasizes the need to validate Western VTE management guidelines in populations from Asia.

\section{Conclusion}

Together, Asian countries account for more than half the world's population and their contribution to the global VTErelated burden of disease is considerable, albeit at lower levels than Western countries. This systematic review reinforces and extends conclusions from previous reviews that VTE rates in Asia are significant and rising, both community-wide and among medical and surgical inpatients. Although the published data have methodological limitations, the evidence is consistent and persuasive regarding the incidence and predispositions for VTE in East Asia.

There is a need for greater awareness of VTE and its diagnosis and management in old age, cancer, inflammatory disorders and after surgery. Advice on anticoagulant or other VTE prophylaxis after surgery in Asia requires more extensive studies of both incidence and potential bleeding risks. Without more local evidence, the local guidelines on VTE prevention and management, which are tailored to needs of the Asia-Pacific region, must rely heavily on North American or European guidance. Doubts about their local applicability may contribute to poor guideline adherence.

\section{What is known on this topic?}

- There tends to be a lack of awareness of venous thromboembolism (VTE) as a problem in Asian populations.

- Although a large number of studies have investigated the incidence of VTE in Asia, there remains a lack of agreement regarding the true present risks of VTE in Asian populations.

\section{What this paper adds?}

- This systematic literature review brings together and summarizes published evidence on VTE from several Asian countries.

- The review examines literature published since 1995 on the incidence of symptomatic VTE (DVT or PE) in Asian populations and includes data from 73 studies.

- Population-wide estimates of annual VTE rates in Korea, Taiwan and Hong Kong (13.8, 15.9 and 19.9 per 100,000 people, respectively) are approximately 15 to $20 \%$ of the level recorded in Western countries ( $\sim 100$ per 100,000 people).

- The reported incidence of VTE in Asia has increased over time.

- These findings highlight the need for awareness that VTE is an important disorder in Asian populations.

\section{Authors' Contributions}

All authors were involved in drafting the article or revising it critically for important intellectual content, and all authors approved the final version to be submitted for publication. Study conception and design: L.H.L., A.G., R.J., C.W., and C.C.W.

Acquisition of data: L.H.L. and A.G.

Analysis and interpretation of data: L.H.L., A.G., R.J., C.W., and C.C.W.

\section{Acknowledgements}

Medical writing support has been provided by Dr. Ruth Hadfield on behalf of MediTech Media Asia Pacific Pte Ltd and was funded by Bristol-Myers Squibb and Pfizer. The sponsors had no involvement in the collection and analysis of publications to be included in this review. Dr. Zhenghuo Zhai has supported Prof. Chen Wang on manuscript development.

\section{References}

1 Roberts LN, Patel RK, Arya R. Venous thromboembolism and ethnicity. Br J Haematol 2009;146(04):369-383

2 Bounameaux H, Rosendaal FR. Venous thromboembolism: why does ethnicity matter? Circulation 2011;123(20):2189-2191 
3 Heit JA. The epidemiology of venous thromboembolism in the community: implications for prevention and management. J Thromb Thrombolysis 2006;21(01):23-29

4 Angchaisuksiri P. Venous thromboembolism in Asia: an unrecognised and under-treated problem? Thromb Haemost 2011;106 (04):585-590

5 Guyatt G, Drummond R, Meade M, et al. The Evidence BasedMedicine Working Group Users' Guides to the Medical Literature. 3rd ed. Chicago, IL: McGraw Hill; 2013

6 Liberati A, Altman DG, Tetzlaff J, et al. The PRISMA statement for reporting systematic reviews and meta-analyses of studies that evaluate healthcare interventions: explanation and elaboration. BMJ 2009;339:b2700

7 Jang MJ, Bang SM, Oh D. Incidence of venous thromboembolism in Korea: from the Health Insurance Review and Assessment Service database. J Thromb Haemost 2011;9(01):85-91

8 Lee CH, Lin LJ, Cheng CL, Kao Yang YH, Chen JY, Tsai LM. Incidence and cumulative recurrence rates of venous thromboembolism in the Taiwanese population. J Thromb Haemost 2010;8(07): 1515-1523

9 Cheuk BL, Cheung GC, Cheng SW. Epidemiology of venous thromboembolism in a Chinese population. Br J Surg 2004;91 (04):424-428

10 Sakuma M, Konno Y, Shirato K. Increasing mortality from pulmonary embolism in Japan, 1951-2000. Circ J 2002;66(12): 1144-1149

11 Chung WS, Lin CL, Chang SN, Lu CC, Kao CH. Systemic lupus erythematosus increases the risks of deep vein thrombosis and pulmonary embolism: a nationwide cohort study. J Thromb Haemost 2014;12(04):452-458

12 Chung WS, Lin CL, Sung FC, et al. Systemic sclerosis increases the risks of deep vein thrombosis and pulmonary thromboembolism: a nationwide cohort study. Rheumatology (Oxford) 2014;53(09): $1639-1645$

13 Chen WJ, Lin CC, Lin CY, et al. Pulmonary embolism in chronic obstructive pulmonary disease: a population-based cohort study. COPD 2014;11(04):438-443

14 Chung WS, Lin CL, Ho FM, et al. Asthma increases pulmonary thromboembolism risk: a nationwide population cohort study. Eur Respir J 2014;43(03):801-807

15 Chen CY, Liao KM. The incidence of deep vein thrombosis in Asian patients with chronic obstructive pulmonary disease. Medicine (Baltimore) 2015;94(44):e1741

16 Chung WS, Lin CL, Sung FC, Hsu WH, Chen YF, Kao CH. Increased risks of deep vein thrombosis and pulmonary embolism in Sjögren syndrome: a nationwide cohort study. J Rheumatol 2014;41(05):909-915

17 Chung WS, Peng CL, Lin CL, et al. Rheumatoid arthritis increases the risk of deep vein thrombosis and pulmonary thromboembolism: a nationwide cohort study. Ann Rheum Dis 2014;73(10): 1774-1780

18 Kang JH, Keller JJ, Lin YK, Lin HC. A population-based case-control study on the association between rheumatoid arthritis and deep vein thrombosis. J Vasc Surg 2012;56(06):1642-1648

19 Chen YG, Lin TY, Huang WY, Lin CL, Dai MS, Kao CH. Association between pneumococcal pneumonia and venous thromboembolism in hospitalized patients: a nationwide population-based study. Respirology 2015;20(05):799-804

20 Lin TY, Chen YG, Huang WY, et al. Association between chronic osteomyelitis and deep-vein thrombosis. Analysis of a nationwide population-based registry. Thromb Haemost 2014;112(03): 573-579

21 Chung WS, Lin CL, Chen YF, Hsu WH, Kao CH. Pulmonary tuberculosis increases the risk of pulmonary thromboembolism: a nationwide population-based cohort study. Thromb Haemost 2014;112(06):1325-1327

22 Wang CC, Chang CT, Lin CL, Lin IC, Kao CH. Hepatitis C virus infection associated with an increased risk of deep vein throm- bosis: a population-based cohort study. Medicine (Baltimore) 2015;94(38):e1585

$23 \mathrm{Ng} \mathrm{KJ}$, Lee YK, Huang MY, Hsu CY, Su YC. Risks of venous thromboembolism in patients with liver cirrhosis: a nationwide cohort study in Taiwan. J Thromb Haemost 2015;13(02):206-213

24 Chung WS, Lin CL, Chang SN, Chung HA, Sung FC, Kao CH. Increased risk of deep vein thrombosis and pulmonary thromboembolism in patients with spinal cord injury: a nationwide cohort prospective study. Thromb Res 2014;133(04):579-584

25 Lim YP, Lin CL, Hung DZ, Ma WC, Lin YN, Kao CH. Increased risk of deep vein thrombosis and pulmonary thromboembolism in patients with organophosphate intoxication: a nationwide prospective cohort study. Medicine (Baltimore) 2015;94(01):e341

26 Chung WS, Lin CL, Kao CH. Carbon monoxide poisoning and risk of deep vein thrombosis and pulmonary embolism: a nationwide retrospective cohort study. J Epidemiol Community Health 2015; 69(06):557-562

27 Hsu WY, Lane HY, Lin CL, Kao CH. A population-based cohort study on deep vein thrombosis and pulmonary embolism among schizophrenia patients. Schizophr Res 2015;162(1-3):248-252

28 Chung WS, Chen YF, Lin CL, Chang SN, Hsu WH, Kao CH. Sleep disorders increase the risk of venous thromboembolism in individuals without sleep apnea: a nationwide population-based cohort study in Taiwan. Sleep Med 2015;16(01):168-172

29 Lee $\mathrm{CH}$, Cheng CL, Kao Yang YH, Lin LJ. Hormone therapy and risk of venous thromboembolism among postmenopausal women in Taiwan: a 10-year nationwide population-based study. Circ J 2015;79(05):1107-1114

30 Chung WS, Lin CL, Kao CH. Diabetes increases the risk of deep-vein thrombosis and pulmonary embolism. A population-based cohort study. Thromb Haemost 2015;114(04):812-818

31 Lu DY, Huang CC, Huang PH, et al. Metformin use in patients with type 2 diabetes mellitus is associated with reduced risk of deep vein thrombosis: a non-randomized, pair-matched cohort study. BMC Cardiovasc Disord 2014; 14:187

32 Wu PK, Chen CF, Chung LH, Liu CL, Chen WM. Population-based epidemiology of postoperative venous thromboembolism in Taiwanese patients receiving hip or knee arthroplasty without pharmacological thromboprophylaxis. Thromb Res 2014;133 (05):719-724

33 Lee $\mathrm{CH}$, Cheng $\mathrm{CL}$, Chang $\mathrm{CH}$, et al. Universal pharmacological thromboprophylaxis for total knee arthroplasty may not be necessary in low-risk populations: a nationwide study in Taiwan. J Thromb Haemost 2012;10(01):56-63

34 Lee CH, Lin TC, Cheng CL, Yang CY, Lin LJ, Kao Yang YH. Comparative risk of venous thromboembolism between total knee and hip replacement: a 10-year nationwide population-based study in Taiwan. J Thromb Haemost 2013;11(10):1930-1932

35 Masuda K, Chikuda H, Yasunaga H, et al. Factors affecting the occurrence of pulmonary embolism after spinal surgery: data from the national administrative database in Japan. Spine J 2012; 12(11):1029-1034

36 Yhim HY, Jang MJ, Bang SM, et al. Incidence of venous thromboembolism following major surgery in Korea: from the Health Insurance Review and Assessment Service database. J Thromb Haemost 2014;12(07):1035-1043

37 Lee SY, Ro H, Chung CY, et al. Incidence of deep vein thrombosis after major lower limb orthopedic surgery: analysis of a nationwide claim registry. Yonsei Med J 2015;56(01):139-145

38 Lee J, Kim SC, Kim SJ, et al. Prevention of venous thromboembolism in medical intensive care unit: a multicenter observational study in Korea. J Korean Med Sci 2014;29(11):1572-1576

39 Lee S, Hwang JI, Kim Y, Yoon PW, Ahn J, Yoo JJ. Venous thromboembolism following hip and knee replacement arthroplasty in Korea: a nationwide study based on claims registry. J Korean Med Sci 2016;31(01):80-88

40 Jang MJ, Bang SM, Oh D. Incidence of pregnancy-associated venous thromboembolism in Korea: from the Health Insurance 
Review and Assessment Service database. J Thromb Haemost 2011;9(12):2519-2521

41 Yang Y, Liang L, Zhai Z, et al; Investigators for National Cooperative Project for Prevention and Treatment of PTE-DVT. Pulmonary embolism incidence and fatality trends in Chinese hospitals from 1997 to 2008: a multicenter registration study. PLoS One 2011;6 (11):e26861

42 Zhou X, Qian W, Li J, et al. Who are at risk for thromboembolism after arthroplasty? A systematic review and meta-analysis. Thromb Res 2013;132(05):531-536

43 Lee AD, Stephen E, Agarwal S, Premkumar P. Venous thromboembolism in India. Eur J Vasc Endovasc Surg 2009;37(04): 482-485

44 Kishimoto M, Lim HY, Tokuda Y, et al. Prevalence of venous thromboembolism at a teaching hospital in Okinawa, Japan. Thromb Haemost 2005;93(05):876-879

45 Choi WI, Lee MY, Oh D, Rho BH, Hales CA. Estimated incidence of acute pulmonary embolism in a Korean hospital. Clin Appl Thromb Hemost 2011;17(03):297-301

46 Lee LH, Gu KQ Heng D. Deep vein thrombosis is not rare in Asiathe Singapore General Hospital experience. Ann Acad Med Singapore 2002;31(06):761-764

$47 \mathrm{Ng} \mathrm{HJ}$, Lee LH. Trends in prevalence of deep venous thrombosis among hospitalised patients in an Asian institution. Thromb Haemost 2009;101(06):1095-1099

48 Molina JA, Jiang ZG, Heng BH, Ong BK. Venous thromboembolism at the National Healthcare Group, Singapore. Ann Acad Med Singapore 2009;38(06):470-478

49 Yang Y, Zhang XZ, Ng HS, Fong JC, Lee LH. The effect of chronic liver disease on venous thromboembolism among medically managed patients in Singapore General Hospital. Thromb Res 2015;136 (03):548-551

50 Kunisawa S, Ikai H, Imanaka Y. Incidence and prevention of postoperative venous thromboembolism: are they meaningful quality indicators in Japanese health care settings? World J Surg 2012;36(02):280-286

$51 \mathrm{Yu} \mathrm{YB}$, Gau JP, Liu CY, et al. A nation-wide analysis of venous thromboembolism in 497,180 cancer patients with the development and validation of a risk-stratification scoring system. Thromb Haemost 2012;108(02):225-235

52 Chew TW, Gau CS, Wen YW, Shen LJ, Mullins CD, Hsiao FY. Epidemiology, clinical profile and treatment patterns of venous thromboembolism in cancer patients in Taiwan: a populationbased study. BMC Cancer 2015;15:298

53 Lee YG, Kim I, Lee E, et al. Risk factors and prognostic impact of venous thromboembolism in Asian patients with non-small cell lung cancer. Thromb Haemost 2014;111(06):1112-1120

54 Wang Z, Yan HH, Yang JJ, et al. Venous thromboembolism risk factors in Chinese non-small cell lung cancer patients. Support Care Cancer 2015;23(03):635-641

55 Tsai SJ, Ruan YX, Lee CC, et al. The incidence of venous thromboembolism in cervical cancer: a nationwide population-based study. BMC Res Notes 2012;5:316

56 Oranratanaphan S, Termrungruanglert W, Khemapech N. Incidence and clinical characteristic of venous thromboembolism in gynecologic oncology patients attending King Chulalongkorn Memorial Hospital over a 10 year period. Asian Pac J Cancer Prev 2015;16(15):6705-6709

57 Kang MJ, Ryoo BY, Ryu MH, et al. Venous thromboembolism (VTE) in patients with advanced gastric cancer: an Asian experience. Eur J Cancer 2012;48(04):492-500

58 Kato A, Takano H, Ichikawa A, et al. A retrospective cohort study of venous thromboembolism(VTE) in 1035 Japanese myeloma patients treated with thalidomide; lower incidence without statistically significant association between specific risk factors and development of VTE and effects of thromboprophylaxis with aspirin and warfarin. Thromb Res 2013;131 (02):140-144
59 Lee YG, Kim I, Kwon JH, et al. Implications of cytogenetics for venous thromboembolism in acute myeloid leukaemia. Thromb Haemost 2015;113(01):201-208

60 Sugimura K, Sakuma M, Shirato K. Potential risk factors and incidence of pulmonary thromboembolism in Japan: results from an overview of mailed questionnaires and matched casecontrol study. Circ J 2006;70(05):542-547

61 ISTH Steering Committee for World Thrombosis Day. Thrombosis: a major contributor to the global disease burden. J Thromb Haemost 2014;12(10):1580-1590

62 Cohen AT, Agnelli G, Anderson FA, et al; VTE Impact Assessment Group in Europe (VITAE). Venous thromboembolism (VTE) in Europe. The number of VTE events and associated morbidity and mortality. Thromb Haemost 2007;98(04):756-764

63 Tamariz L, Harkins T, Nair V. A systematic review of validated methods for identifying venous thromboembolism using administrative and claims data. Pharmacoepidemiol Drug Saf 2012;21(Suppl 1):154-162

64 Xin G, Wei Z, Ji C, et al. Metformin uniquely prevents thrombosis by inhibiting platelet activation and mtdna release. Sci Rep 2016; 6:36222

65 Heit JA, Mohr DN, Silverstein MD, Petterson TM, O'Fallon WM, Melton LJ III. Predictors of recurrence after deep vein thrombosis and pulmonary embolism: a population-based cohort study. Arch Intern Med 2000;160(06):761-768

66 Wendelboe AM, McCumber M, Hylek EM, Buller H, Weitz JI, Raskob G; ISTH Steering Committee for World Thrombosis Day. Global public awareness of venous thromboembolism. J Thromb Haemost 2015;13(08):1365-1371

67 Chan LY, Tam WH, Lau TK. Venous thromboembolism in pregnant Chinese women. Obstet Gynecol 2001;98(03):471-475

68 Liew NC, Alemany GV, Angchaisuksiri P, et al. Asian venous thromboembolism guidelines: updated recommendations for the prevention of venous thromboembolism. Int Angiol 2017; 36(01):1-20

69 Wang KL, Chu PH, Lee $\mathrm{CH}$, et al. Management of venous thromboembolisms: part I. The consensus for deep vein thrombosis. Acta Cardiol Sin 2016;32(01):1-22

70 Bang SM, Jang MJ, Kim KH, et al. Prevention of venous thromboembolism, 2nd edition: Korean Society of Thrombosis and Hemostasis Evidence-based Clinical Practice Guidelines. J Korean Med Sci 2014;29(02):164-171

71 Cohen AT; Asia-Pacific Thrombosis Advisory Board. Asia-Pacific Thrombosis Advisory Board consensus paper on prevention of venous thromboembolism after major orthopaedic surgery. Thromb Haemost 2010;104(05):919-930

72 JCS Joint Working Group. Guidelines for the diagnosis, treatment and prevention of pulmonary thromboembolism and deep vein thrombosis (JCS 2009). Circ J 2011;75(05):1258-1281

73 Parakh R, Kakkar VV, Kakkar AK; Venous Thromboembolism (VTE) Core Study Group. Management of venous thromboembolism. J Assoc Physicians India 2007;55:49-70

74 Rahman ARA, Sathar J, Chee CC, et al. Malaysian Clinical Practice Guidelines: Prevention and Treatment of Venous Thromboembolism. Available at: http://www.moh.gov.my/penerbitan/CPG2017/9005. pdf. Accessed May 23, 2017

75 ST LI T, Working Group for the Prevention of Venous Thromboembolism in ICU in Hong Kong, HKSCCM. Prevention of Venous Thromboembolism in Intensive Care Units in Hong Kong. Available at: http://hksccm.org/index.php/professional/133-clinicalmanagement/1756-prevention-of-venous-thromboembolismin-icu-in-hong-kong. Accessed Oct 25, 2017

76 Chung WS, Lin CL, Hsu WH, Kao CH. Inflammatory bowel disease increases the risks of deep vein thrombosis and pulmonary embolism in the hospitalized patients: a nationwide cohort study. Thromb Res 2015;135(03):492-496

77 Chung WS, Lin CL, Sung FC, Lu CC, Kao CH. Increased risk of venous thromboembolism in patients with dermatomyositis/polymyositis: a nationwide cohort study. Thromb Res 2014;134(03):622-626 
78 Wang CC, Lin CL, Wang GJ, Chang CT, Sung FC, Kao CH. Atrial fibrillation associated with increased risk of venous thromboembolism. A population-based cohort study. Thromb Haemost 2015; 113(01):185-192

79 Zhou HX, Peng LQ, Yan Y, et al. Validation of the Caprini risk assessment model in Chinese hospitalized patients with venous thromboembolism. Thromb Res 2012;130(05):735-740

80 Aniwan S, Rojnuckarin P. High incidence of symptomatic venous thromboembolism in Thai hospitalized medical patients without thromboprophylaxis. Blood Coagul Fibrinolysis 2010;21(04): 334-338

81 Rojnuckarin P, Uaprasert N, Vajragupta L, Numkarunarunrote N, Tanpowpong N, Sutcharitchan P. Risk factors for symptomatic venous thromboembolism in Thai hospitalised medical patients. Thromb Haemost 2011;106(06):1103-1108

82 Chen CC, Lee TH, Chung CY, et al. Symptomatic pulmonary embolism among stroke patients in Taiwan: a retrospective cohort study. Top Stroke Rehabil 2012;19(05):361-368

83 Wong TH, Koh MP, Ng J. Symptomatic venous thromboembolism in Asian major trauma patients: incidence, presentation and risk factors. Eur J Trauma Emerg Surg 2013;39(05):495-500

84 Won MH, Lee GW, Lee TJ, Moon KH. Prevalence and risk factors of thromboembolism after joint arthroplasty without chemical thromboprophylaxis in an Asian population. J Arthroplasty 2011;26(07):1106-1111

85 Wu PK, Chen CF, Chung LH, Liu CL, Chen WM. Pulmonary embolism following total knee arthroplasty in Taiwanese patients. J Chin Med Assoc 2014;77(03):155-159

86 Leizorovicz A, Turpie AG, Cohen AT, Wong L, Yoo MC, Dans A; SMART Study Group. Epidemiology of venous thromboembolism in Asian patients undergoing major orthopedic surgery without thromboprophylaxis. The SMART study. J Thromb Haemost 2005; 3(01):28-34

87 Park YG, Ha CW, Lee SS, Shaikh AA, Park YB. Incidence and fate of 'Symptomatic' venous thromboembolism after knee arthroplasty without pharmacologic prophylaxis in an Asian population. J Arthroplasty 2016;31(05):1072-1077

88 Tsuda Y, Yasunaga H, Horiguchi H, Fushimi K, Kawano H, Tanaka S. Effects of fondaparinux on pulmonary embolism following hemiarthroplasty for femoral neck fracture: a retrospective observational study using the Japanese Diagnosis Procedure Combination database. J Orthop Sci 2014;19(06):991-996

89 Chan LY, Yuen PM, Lo WK, Lau TK. Symptomatic venous thromboembolism in Chinese patients after gynecologic surgery: incidence and disease pattern. Acta Obstet Gynecol Scand 2002;81(04):343-346

$90 \mathrm{Qu} \mathrm{H}, \mathrm{Li}$ Z, Zhai Z, et al. Predicting of venous thromboembolism for patients undergoing gynecological surgery. Medicine (Baltimore) 2015;94(39):e1653

91 Yang SS, Yu CS, Yoon YS, Yoon SN, Lim SB, Kim JC. Symptomatic venous thromboembolism in Asian colorectal cancer surgery patients. World J Surg 2011;35(04):881-887

92 Liu HS, Kho BC, Chan JC, et al. Venous thromboembolism in the Chinese population-experience in a regional hospital in Hong Kong. Hong Kong Med J 2002;8(06):400-405

93 Cheng G, Chan C, Liu YT, et al. Incidence of deep vein thrombosis in hospitalized Chinese medical patients and the impact of DVT prophylaxis. Thrombosis 2011;2011:629383

94 Nakamura M, Sakuma M, Yamada N, et al; JaSPER investigators. Risk factors of acute pulmonary thromboembolism in Japanese patients hospitalized for medical illness: results of a multicenter registry in the Japanese society of pulmonary embolism research. J Thromb Thrombolysis 2006;21(02):131-135

\section{Appendix A}

Search string: (("Venous Thromboembolism"[All Fields] OR "Venous Thrombosis”[All Fields]) OR "Pulmonary Embolism”[All Fields]) AND ((“asian continental ancestry group"[MeSH Terms] OR ("asian"[All Fields] AND "continental”[All Fields] AND “ancestry"[All Fields] AND "group”[All Fields]) OR “asian continental ancestry group”[All Fields] OR "asian”[All Fields]) OR “Taiwan”[Mesh] OR “Hong Kong”[Mesh] OR “Singapore”[Mesh] OR “China”[Mesh] OR “Korea”[Mesh] OR “Malaysia”[Mesh] OR “Philippines”[Mesh] OR “Thailand”[Mesh] OR “Japan”[Mesh] OR “India”[Mesh] OR “Indonesia”[Mesh] OR “Ethnic groups”[Mesh]) AND ((“1995/01/29”[PDAT]: “2016/02/23”[PDAT]) AND English[lang]). 